\title{
DIMENSIONALLY NILPOTENT JORDAN ALGEBRAS
}

\author{
J. MARSHALL OSBORN
}

(Communicated by Maurice Auslander)

\begin{abstract}
An algebra $A$ of dimension $n$ is called dimensionally nilpotent if it has a nilpotent derivation $\partial$ with the property that $\partial^{n-1} \neq 0$. Here we show that a dimensionally nilpotent Jordan algebra $A$ over a perfect field of characteristic not 2 or 3 is either (i) nilpotent, or (ii) one-dimensional modulo its maximal nilpotent ideal. This result is also extended to noncommutative Jordan algebras.
\end{abstract}

\section{INTRODUCTION}

The structure of dimensionally nilpotent Lie algebras was studied by Leger and Manley [7] for characteristic 0, and by the author [9-11] for characteristic $p>5$. Here we investigate dimensionally nilpotent Jordan algebras in $\S 2$ and noncommutative Jordan algebras in $\S 3$. We assume throughout that $F$ is a field of characteristic not 2 or 3 .

For our result, we need some information about the derivations of the Jordan algebra $F_{q}^{+}$, where $F_{q}$ is the associative algebra of $q \times q$ matrices over $F$, and $F_{q}^{+}$is the same algebra under the Jordan product $x \circ y=(x y \times y x) / 2$. We recall that the derivations of $F_{q}^{+}$were found for characteristic 0 by Jacobson [4], and for characteristic $p$ with $q$ not divisible by $p$ by Harris [3]. The case when $q$ is divisible by $p$, which is what we need, is a special case of Corollary 4.9 of [1] (see also I. N. Herstein, Topics in Ring Theory, Univ. of Chicago Press, Chicago, 1969, p. 55). Specifically, we require

Proposition 1.1. When $q \geq 3$, the derivations of $F_{q}^{+}$are exactly the derivations of $F_{q}$, namely, the maps of the form $\operatorname{ad}_{x}$ for $x \in F_{q}$ where $\operatorname{ad}_{x} y=[x, y]=$ $x y-y x$.

We also need the following result, which is obvious from the form of $\partial$.

Lemma 1.2. If $B$ and $C$ are two $\partial$-invariant subspaces of a dimensionally nilpotent algebra $A$, then either $B \subset C$ or $C \subset B$.

An algebra is called derivation simple if it contains no proper ideals that are invariant under all derivations.

Received by the editors October 4, 1990 and, in revised form, November 27, 1990 and April $25,1991$.

1980 Mathematics Subject Classification (1985 Revision). Primary 17C30, 17A15.

The author gratefully acknowledges the support of the National Science Foundation under Grant DMS-8800691. 
Proposition 1.3. Let $A$ be a derivation simple dimensionally nilpotent algebra. Then either $A$ is simple, or char $F=p>0$ and $A$ is isomorphic to $S \otimes B_{r}$, where $S$ is a simple dimensionally nilpotent algebra, and $B_{r}$ is the truncated polynomial algebra on $r$ indeterminates.

Proof. Although the proof is virtually the same as the proof of Proposition 2.1 of [9], we give it here for completeness. Suppose that $A$ satisfies the hypothesis, i.e., that $A$ has no proper ideals that are invariant under all derivations. It follows from Block [2] that either $A$ is simple, or the characteristic is $p>0$ and $A \cong S \otimes B_{r}$, where $S$ is a simple Lie algebra and $B_{r}$ is the truncated polynomial algebra on $r$ indeterminates. Writing $S^{\prime}=S \otimes B_{r-1}$, we have $A \cong S^{\prime} \otimes B_{1}$. In order to prove Proposition 1.3 by induction on the dimension of $A$, it is sufficient to establish that $A$ dimensionally nilpotent implies that $S^{\prime}$ is also. If the field $F$ is chosen so that $S$ is central simple, the derivation algebra of $S^{\prime} \otimes B_{1}$ is known [2] to be $\operatorname{Der} S^{\prime} \otimes B_{1}+1 \otimes \operatorname{Der} B_{1}$. In the representation of $\partial$ as an element of $\operatorname{Der} S^{\prime} \otimes B_{1}+1 \otimes \operatorname{Der} B_{1}$, we can think of the element of Der $B_{1}$ as having the form $g(x) \partial_{x}$ for some $g(x) \in B_{1}$ where $\partial_{x}$ is partial differentiation with respect to $x$ in $B_{1}$. We say that a derivation of $A$ is of type 1 if $g(x)$ has a nonzero constant term and of type 2 otherwise.

If $\partial$ is of type 2, then for $s \in S, \partial\left(s \otimes x^{j}\right)$ has no terms of lower degree in $x$ than $j$. Thus, $S^{\prime} \otimes x^{p-1}$ is a $\partial$-invariant subalgebra of $A$. If $\partial$ acts nilpotently with a one-dimensional eigenspace on $A$, then the same is true on $S^{\prime} \otimes x^{p-1}$; hence $S^{\prime}$ is dimensionally nilpotent under the induced action of $\partial$ on $S^{\prime}$.

We may suppose that $\partial$ is of type 1 , so that $\partial=a \partial_{x}+\partial_{2}$ where $a \in F$ is nonzero and where $\partial_{2}\left(S^{\prime} \otimes x^{j}\right) \subset S^{\prime} \otimes x^{j} B_{1}$. For any derivation $\partial^{\prime}$ of type 1 , let $C_{p-1}=S^{\prime} \otimes x^{p-1}$, and define $C_{i}=\partial^{\prime} C_{i+1}$ by downward induction on $i$. Because of the form of a derivation of type 1, no element of $C_{i+1}$ is annihilated by $\partial^{\prime}$ for $i \geq 0$. In fact, no nonzero element of $\sum_{i>0} C_{i}$ is annihilated by $\partial^{\prime}$. Clearly $A$ is the vector space direct sum of the $C_{i}$ 's for $0 \leq i \leq p-1$. The form of the elements of each $C_{i}$ for $i \leq p-2$ depends on the derivation $\partial^{\prime}$, but the space $\sum_{i \geq s} C_{i}$ depends only on $s$ and is independent of the particular derivation of type 1 used to define it.

Let $\partial_{j}$ denote the $p^{j}$ power of our derivation $\partial$, and let $r$ be the largest integer such that $p^{r}<\operatorname{dim} A$. Then $\partial_{r+1}=0$ is of type 2 . Since we are assuming that $\partial_{0}=\partial$ is of type 1 , there must exist an integer $k$ such that $\partial_{k}$ is of type 1 and $\partial_{k+1}$ is of type 2. Then, defining the spaces $C_{i}$ for the derivation $\partial_{k}$, we have $\partial_{k+1} C_{p-1} \subset C_{p-1}$; so $\partial_{k} C_{0}=\left(\partial_{k}\right)^{p} C_{p-1} \subset C_{p-1}$. If $u=u_{0}+u_{1}+\cdots+u_{p-1} \in \operatorname{Ker} \partial_{k}$, then $0=\partial_{k} u_{0}+\partial_{k} u_{1}+\cdots+\partial_{k} u_{p-1}$, which implies that $0=u_{1}=u_{2}=\cdots=u_{p-1}$ and that $u=u_{0} \in \operatorname{Ker} \partial_{k}$. Hence, $\operatorname{dim} \operatorname{Ker} \partial_{k}=p^{k} \leq \operatorname{dim} S^{\prime}$.

Suppose first that $k>1$, and let $C_{0}^{\prime}, \ldots, C_{p-1}^{\prime}$ be the $C_{i}$ 's determined by $\partial$. Then an element $w \in A$ which is not in $\partial A$ is not in $\sum_{i \neq p-1} C_{i}^{\prime}$, and so can be taken to be in $C_{p-1}^{\prime}=C_{p-1}$. If $n=\operatorname{dim} A$, the span of the elements of the form $\partial^{n-j} w$ for $1 \leq j \leq p^{k}$ is exactly the roots of $\partial_{k}$, and they are all in $C_{0}$ by the last paragraph. Hence the span $G$ of the elements of the form $\partial^{n-j} w$ for $p^{k}+1 \leq j \leq 2 p^{k}$ is contained in $C_{1} \subset \sum_{i>0} C_{i}=\sum_{i>0} C_{i}^{\prime}$. In fact, each of the elements $\partial^{l} w$ spanning $G$ has a nonzero component in $C_{1}^{\prime}$. But 
then $\partial^{l+1} w=\partial\left(\partial^{l} w\right) \notin \sum_{i>0} C_{i}$, which is a contradiction since $\operatorname{dim} G>1$. This rules out the case $k>0$.

When $k=1$, we have $\partial^{n-i} w \in C_{i-1}$ for $1 \leq i \leq p$, from which it is clear that $\operatorname{dim} C_{p-1} \cap \operatorname{Ker} \partial_{1}=1$. Then the action of $\partial_{1}$ on $C_{p-1}$ makes $S^{\prime}$ into a dimensionally nilpotent Lie algebra using the nilpotent derivation $\partial^{\prime}$ on $S^{\prime}$ induced by $\partial_{1}$.

Lemma 1.4. Let $A$ be a simple power associative algebra of dimension greater than one containing an identity element 1. Then $\operatorname{char} F=p>0$ and $\operatorname{dim} A=$ $p^{r}$ for some positive integer $r$, and $A$ contains an element $z$ with $z^{p}=0$ and $z^{p-1} \neq 0$.

Proof. Since 1 is annihilated by all derivations, it spans the one-dimensional subspace of $A$ annihilated by $\partial$. Let $z_{1}$ be such that $\partial\left(z_{1}\right)=1$. Then $\partial\left(z_{1}^{i}\right)=$ $i z_{1}^{i-1}$, showing that $z_{1}^{i} \neq 0$ if $i z_{1}^{i-1} \neq 0$. If $\operatorname{char} F=0$, then the powers of $z_{1}$ are linearly independent and the dimension of the subalgebra generated by $z_{1}$ must be infinite, contrary to the hypothesis. Thus, char $F=p>0$, and we see that $1, z_{1}, z_{1}^{2}, \ldots, z_{1}^{p-1}$ are linearly independent. Since $\partial\left(z_{1}^{p}\right)=0$, it follows that $z_{1}^{p}$ is a multiple of 1 . After subtracting an appropriate multiple of 1 from $z_{1}$, we can suppose that $z_{1}^{p}=0$.

Now suppose that $z_{1}, z_{2}, \ldots, z_{k}$ are given, and $0 \leq i \leq p^{k}-1$, that $z^{\langle i\rangle}=z_{1}^{i_{1}} z_{2}^{i_{2}} \cdots z_{k}^{i_{k}}$ where the products of powers are associated from the left end, and where $i=i_{1}+i_{2} p+\cdots+i_{k} p^{k-1}$ is the expansion of $i$ in the base $p$. Let $B_{k}^{\prime}$ be the span of the $z^{\langle i\rangle}$ with $0 \leq i \leq p^{k}-1$. Assume inductively that $\partial\left(z^{\langle i\rangle}\right)$ is a nonzero multiple of $z^{\langle i-1\rangle}$ modulo $z$ 's with lower superscripts, and that $B_{k}^{\prime}$ is a subalgebra of $A$. If $B_{k}^{\prime} \neq A$, and if $q=p^{k}$, then there exists an element $z_{k+1}$ such that $\partial\left(z_{k+1}\right)=z^{\langle q-1\rangle}$. Thus, $\partial^{q}\left(z_{k+1}\right)=a 1$ for some nonzero $a \in F$, and $\partial^{q}\left(z_{k+1}^{i}\right)=i a z_{k+1}^{i-1}$, from which we see that $z_{k+1}^{i} \neq 0$ for $1 \leq i \leq p-1$ and that $z_{k+1}^{p} \in B_{k}^{\prime}$. Extend the definition of $z^{\langle i\rangle}$ by letting $z^{\langle i\rangle}=z_{1}^{i_{1}} z_{2}^{i_{2}} \cdots z_{k+1}^{i_{k+1}}$ for $0 \leq i \leq p^{k+1}-1$, where $i=i_{1}+i_{2} p+\cdots+i_{k+1} p^{k}$ is the expansion of $i$ in the base $p$. Let $B_{k+1}^{\prime}$ be the span of the $z^{\langle i\rangle}$ for $0 \leq i \leq p^{k+1}-1$.

To complete the inductive step, we must show for $0 \leq i \leq p^{k+1}-1$ that $\partial\left(z^{\langle i\rangle}\right)$ is a nonzero multiple of $z^{\langle i-1\rangle}$ modulo lower terms, and that $B_{k+1}^{\prime}$ is a subalgebra. We establish the first by induction on $i$, noting that it is assumed to be true when $i<p^{k}$ :

$$
\begin{aligned}
\partial^{q} \partial\left(z^{\langle i\rangle}\right) & =\partial\left[\partial^{q}\left(z_{1}^{i_{1}} z_{2}^{i_{2}} \cdots z_{k}^{i_{k}}\right) z_{k+1}^{i_{k+1}}+\left(z_{1}^{i_{1}} z_{2}^{i_{2}} \cdots z_{k}^{i_{k}}\right) \partial^{q}\left(z_{k+1}^{i_{k+1}}\right)\right] \\
& =\partial\left[\left(z_{1}^{i_{1}} z_{2}^{i_{2}} \cdots z_{k}^{i_{k}}\right) i_{k+1} a z_{k+1}^{i_{k+1}-1}\right]=i_{k+1} a \partial\left(z^{\langle i-q\rangle}\right)
\end{aligned}
$$

Then $B_{k+1}^{\prime}$ can be characterized by the fact that it is the set of elements of $A$ that are annihilated by $\partial^{q p}$, from which it follows that $B_{k+1}^{\prime}$ is a subalgebra.

We have shown by induction that each $B_{k}^{\prime}$ is a subalgebra, and that if $B_{k}^{\prime} \neq A$ then $B_{k+1}^{\prime}$ exists inside $A$. Thus, $A=B_{k}^{\prime}$ for some $k$. Clearly, $\operatorname{dim} B_{k}^{\prime}=p^{k}$, so that $\operatorname{dim} A=p^{k}$. 


\section{The JoRdan CASE}

We can now establish

Theorem 2.1. If $A$ is a dimensionally nilpotent Jordan algebra over a perfect field $F$ with char $F \neq 2,3$, then either $A$ is nilpotent or $\operatorname{dim}(A / \operatorname{Rad} A)=1$.

Proof. If $E$ is the algebraic closure of $F$, then $A_{E}=A \otimes_{F} E$ is also dimensionally nilpotent. Further, if $A_{E}$ satisfies the conclusion of Theorem 2.1 , then so does $A$. Thus it is sufficient to establish Theorem 2.1 in the case when $F$ is algebraically closed. We may also suppose that the maximal $\partial$-invariant nilpotent ideal $N$ of $A$ has been divided out, since $A$ satisfies the conclusion of the theorem if $A / N$ does. Then $A$ is a direct sum of $\partial$-invariant algebras by Block [2] (or Ravisankar [13]). It follows from Lemma 1.2 that $A$ is $\partial$-simple. In view of Proposition 1.3, we need only consider the case when $A$ is simple.

Thus it remains to show that if $A$ is simple then $\operatorname{dim} A=1$. Since simple Jordan algebras have identity elements, the hypotheses of Proposition 1.4 are satisfied. Then, if $\operatorname{dim} A>1$, char $F=p>0$ and $A$ contains an element $z$ that is nilpotent of order exactly $p$. Now, $A$ can have no such element if $A$ is a simple algebra determined by a symmetric bilinear form, or if $A$ is exceptional simple. If $A \cong H\left(F_{q}^{+}, \tau\right)$ for some involution $\tau$ of $F_{q}^{+}$, then $\operatorname{dim} A$ is either $q(q-1) / 2$ or $q(q+1) / 2$, depending on the type of $\tau$. But $\operatorname{dim} A$ is a power of $p$ by Proposition 4.1 , and neither of these numbers can be a prime power. It follows that $A$ has the form $F_{q}^{+}$, where $q$ is a power of $p$. Then the nilpotent derivation $\partial$ on $A$ with the property that $\partial^{n-1} \neq 0$ must have the form $\partial=\operatorname{ad}_{x}$ for some $x \in A$ by Proposition 1.1. Noting that $\partial(x)=0=\partial(1)$, and that the subspace annihilated by $\partial$ is one-dimensional since $\partial^{n-1} \neq 0$, we see that $x$ is a multiple of 1 . But then $\partial(A)=0$, implying that $n=1$.

\section{The NONCOMMUTATIVE JoRdAN CASE}

Here we establish

Theorem 3.1. Let $A$ be a dimensionally nilpotent noncommutative Jordan algebra over a perfect field of characteristic not 2 or 3. Then, modulo its maximal nil ideal, $A$ is either zero-dimensional, one-dimensional, or a nodal algebra.

Proof. Let $\partial$ be a dimensionally nilpotent derivation of $A$. If $A$ has a $\partial$ invariant nil ideal $N$, and if $A / N$ satisfies the conclusion of the theorem, then so does $A$. Thus we may suppose that $A$ is $\partial$-semisimple. By Block [2], $A$ is a direct sum of $\partial$-simple algebras, so that $A$ is $\partial$-simple by Lemma 1.2. It follows from Proposition 1.3 that either $A$ is simple or char $F=p$ and $A \cong B_{r}$. Since the latter case satisfies the conclusion of Theorem 3.1 , we may suppose that $A$ is simple. If $A$ (after possibly making a scalar extension) has two orthogonal idempotents, then so does the Jordan algebra $A^{+}$. But $\partial$ is also a dimensionally nilpotent derivation of $A^{+}$, and so $A^{+}$cannot have two orthogonal idempotents by Theorem 2.1. It follows [14, p. 143] that $A$ must be either one-dimensional or a nodal algebra. 


\section{REFERENCES}

1. G. M. Benkart and J. M. Osborn, Derivations and automorphisms of nonassociative matrix algebras, Trans. Amer. Math. Soc. 263 (1981), 411-430.

2. R. E. Block, Determination of the differentially simple rings with a minimal ideal, Ann. of Math. (2) 90 (1969), 433-459.

3. Bruno Harris, Derivations of Jordan algebras, Pacific J. Math. 9 (1959), 495-512.

4. N. Jacobson, General representation theory of Jordan algebras, Trans. Amer. Math. Soc. 70 (1951), 509-530.

5. L. A. Kokoris, Simple nodal noncommutative Jordan algebras, Proc. Amer. Math. Soc. 9 (1958), 544-550.

6. __ Nodal noncommutative Jordan algebras, Canad. J. Math. 12 (1960), 488-492.

7. G. F. Leger and P. L. Manley, Dimensionally nilpotent Lie algebras, J. Algebra 117 (1988), 162-164.

8. R. H. Oehmke, On Flexible algebras, Ann. of Math. (2) 68 (1958), 221-230.

9. J. M. Osborn, Dimensionally nilpotent Lie algebras of prime characteristic, J. Algebra (to appear).

10. __ Examples of dimensionally nilpotent Lie algebras of prime characteristic, Algebras Groups Geom. 6 (1989), 361-381.

11. __ Radicals of dimensionally nilpotent Lie algebras, submitted.

12. T. S. Ravisankar, A note on a theorem of Kokoris, Proc. Amer. Math. Soc. 21 (1969), 355-356.

13. __ On differentiably simple algebras, Pacific J. Math. 33 (1970), 725-735.

14. R. D. Schafer, An introduction to nonassociative algebra, Academic Press, New York, 1966.

Department of Mathematics, University of Wisconsin, Madison, Wisconsin 53706 